\title{
Effect of Probiotic "L.Reuteri" Association on the Reduction of Serum Bilirubin in Neonatal Jaundice
}

\author{
G. Makhoul, \\ Kobayat Hospital, Kobayat, Lebanon \\ Haykal Hospital, Tripoli, Lebanon
}

J. Mardini,

Faculty of medicine, Holy Spirit University of Kaslik, Kaslik,

UH-Notre Dame Des Secours, Byblos, Lebanon

M. Al Ojaimi,

Balamand University, Koura, Lebanon

G. AbiFares,

Faculty of medicine, Holy Spirit University of Kaslik, Kaslik,

UH-Notre Dame Des Secours, Byblos, Lebanon

P. Hanna,

A. Alameddine,

Haykal Hospital, Tripoli, Lebanon

\author{
H. Feghali, \\ P. Makhoul, \\ J. Saaybi, \\ G. Nicolas, \\ J. Sakkal,
}

M. C. Fadous Khalife,

Faculty of medicine, Holy Spirit University of Kaslik, Kaslik,

UH-Notre Dame Des Secours, Byblos, Lebanon

Doi: 10.19044/esj.2018.v14n12p384 URL:http://dx.doi.org/10.19044/esj.2018.v14n12p384

\begin{abstract}
Objective: Evaluate the effect of probiotics association in reducing the total bilirubin level in the serum of neonates with jaundice. Methods: 69 neonates with indirect hyperbilirubinemia were divided randomly into two groups: control and treatment. The control group was treated using phototherapy and the treatment group was treated using phototherapy plus L.Reuteri probiotic. Inclusion criteria: all term newborns admitted for phototherapy for unconjugated hyperbilirubinemia. Exclusion criteria: septic or ill newborn, phenobarbital therapy, transfusion and parents 'refusal to enter
\end{abstract}


the study. Baseline bilirubin level was obtained prior to initiating phototherapy and then daily for an average of 3 days. Results: Before treatment, the level of bilirubin was similar in the two groups $(\mathrm{p}>0.05)$. We noted a more significant difference in bilirubin at day $1(\mathrm{p}=0.000)$, day $2(=0.000)$ and day $3(\mathrm{p}=0.000)$ during treatment in the probiotic group when compared to the control group. We also noticed a more significant decrease in bilirubin between day 1 and day $2(p=0.000)$ and between day 2 and day $3(p=0.000)$ in the probiotic group when compared to the control group. Conclusion: The decrease of bilirubin in neonates with jaundice is more rapid and more significant in the group receiving probiotics as an adjuvant to phototherapy in case of presence of incompatibility or not.

Keywords: Jaundice; neonate; probiotic; bilirubin; hyperbilirubinemia

\section{Introduction}

Jaundice is one of the most commonly encountered symptom during the neonatal period. It manifests itself as a yellow pigmentation of the mucosa due to an accumulation of bilirubin.(Porter and Dennis 2002)

Hyperbilirubinemia in newborns mostly occurs due to high levels of unconjugated bilirubin in the blood that can have multiple causes such as $\mathrm{ABO} /$ Rhesus incompatibility, infections, prematurity, hypothyroidism, polyglobulia, G6PD deficiency and metabolic disorders. Even unconjugated hyperbilirubinemia is mainly a transitional phenomenon; it may lead to irreversible neurological complications known as "kernicterus". (Alcayd 2008) (Mutombo et al. 2014) Treatment of jaundice requires the treatment of the underlying cause besides the different strategies to decrease serum bilirubin. Phototherapy, intravenous immunoglobulins, and exchange transfusion are the most frequently used therapies due to their efficiency and efficacy in deceasing serum bilirubin with very few side effects. Other therapies include Chinese herbs that activate the hepatic receptor CAR (Huang, Zhang, and Moore 2004), Vimala massages which improves physiological jaundice by increasing newborn defecation (Seyyedrasooli et al. 2014)and enzyme inductors like phenobarbital(Crigler and Gold 1969) . Recently, probiotics are being studied for their potential benefit in being an adjunctive treatment of indirect hyperbilirubinemia (Wenbin Liu 2015).

Probiotics are live microorganisms, which, if given in the right doses, can be beneficial to their host. They are known for their ability to promote the growth of other microorganisms and to increase the activity of bacteria in the intestinal flora. They also play a role in modulating the immune system and producing anti-microorganism substances. (Torkaman et al. 2016) (S et al. 2013) These multiple mechanisms of action of probiotics have made them an object of study with regard to many different pathologies in which they have 
had a proven efficacy, such as in antibiotic-associated diarrhea,(Issa and Moucari 2014) necrotizing enterocolitis,(Gogineni, Morrow, and Malesker 2013) (Chang et al. 2017) (Silverman, Konnikova, and Gerber 2017) allergic reactions(Harmsen 2016) (Cuello-Garcia et al. 2015) (Forsberg et al. 2013), inflammatory bowel disease(Kwon and Farrell 2003), irritable bowel syndrome(Cui and Hu 2012), infantile colic(Mi et al. 2015) (Partty et al. 2015) (Roberts, Ostapchuk, and O'Brien 2004) and neonatal jaundice(W., H., and T. 2015) (Demirel et al. 2013) (Torkaman et al. 2016).

In our study, we compared two treatment modalities of neonatal jaundice: Exclusive phototherapy versus phototherapy combined with L.Reuteri probiotic administration. Our objective was to determine whether serum bilirubin decreases more rapidly with the added probiotic, which would decrease the duration of hospitalization of newborns with jaundice.

\section{Methods \& Statistics}

69 neonates with jaundice from the NICUs of 3 different centers: UHNotre Dame de Secours hospital center, Haykal hospital and Kobayyat hospital were selected for the study between June 2016 and July 2017 after their parents signed a written consent form. The method of selection was by coin tossing.

Inclusion criteria were all term newborns admitted for phototherapy for indirect hyperbilirubinemia

Exclusion criteria were sepsis, newborns receiving IVIG or phenobarbital, or those whose parents refused to give consent to the study.

The neonates were randomized into two groups: Group 1 (control group) included 29 newborns with jaundice who received exclusive phototherapy, while group 2 (treatment group) included 40 newborns who received phototherapy and an adjuvant probiotic "Bio Gaia" containing Lactobacillus Reuteri which was given orally or via bottle once daily ( 100 million live LR per 5 drops).

Venous blood was drawn before treatment and daily during treatment to detect serum bilirubin levels. A complete blood workup was also conducted in order to determine the cause of jaundice in each case.

A form was filled for each child noting their gestational age, birth and actual weight, cause of jaundice, age at the time of treatment plus total serum bilirubin before and on each day during the treatment. A section was reserved at the bottom of the form to report any adverse effects due to the probiotic given.

\section{Analysis}

A statistical analysis was conducted using SPSS version 22 with a confidence interval of $95 \%$. Quantitative data was expressed by means and 
standard deviation while qualitative data was expressed as a percentage. A paired $\mathrm{T}$ test was used for the comparison of means; $\mathrm{p}$ value $<0.05$ was considered significant, $\mathrm{p}$ value $<0.01$ was considered highly significant, and $\mathrm{p}$ value of $<0.001$ was considered extremely significant.

Group 1- the control group- consisted of 29 newborns with an initial total serum bilirubin varying between $11.9 \mathrm{mg} / \mathrm{dl}$ and $24.4 \mathrm{mg} / \mathrm{dl}$ with a mean duration of hospitalization of 3.62 days. Group 2 - the intervention groupconsisted of 40 newborns with an initial total serum bilirubin varying between $11 \mathrm{mg} / \mathrm{dl}$ and $24.6 \mathrm{mg} / \mathrm{dl}$ and a mean duration of hospitalization of 3.4 days.

\section{Results}

The mean of the total serum bilirubin (TSB) measured each day in both groups was calculated:

On day 1 , the mean TSB was $15.55 \mathrm{mg} / \mathrm{dl}$ in-group 1 and $13.7 \mathrm{mg} / \mathrm{dl}$ in-group 2. Day 2, the mean TSB was $12.77 \mathrm{mg} / \mathrm{dl}$ in-group 1 and $11.7 \mathrm{mg} / \mathrm{dl}$ in-group 2 .Day 3 the mean TSB was $11.43 \mathrm{mg} / \mathrm{dl}$ in-group 1 and $11.5 \mathrm{mg} / \mathrm{dl}$ in-group 2.

A comparison of the difference in the mean of total serum bilirubin (TSB) between day 0 and day 1, 2, 3 after treatment was made in each group using the paired $\mathrm{T}$ test. In the probiotic group, results showed a significant drop in total serum bilirubin between day 0 and day $1(p=0.000)$, day 0 and day $2(p=0.000)$ and between day 0 and day $3(p=0.000)$. The decrease in the TSB was very significant between day 1 and day $2(p=0.000)$ and between day 2 and day $3(\mathrm{p}=0.030)$ (Table 1$)$.

Table 1: Variation of the difference in mean TSB in the intervention group.

\begin{tabular}{|c|c|c|}
\hline & $\begin{array}{c}\text { Mean of the difference } \\
\text { in TSB }\end{array}$ & Valeur P \\
\hline Day 0-Day 1 & 5.107 & $\mathbf{0 . 0 0 0}$ \\
\hline Day 0-Day 2 & 7.27 & $\mathbf{0 . 0 0 0}$ \\
\hline Day 0-Day 3 & 7.27 & $\mathbf{0 . 0 0 0}$ \\
\hline Day 1-Day 2 & 2.34 & $\mathbf{0 . 0 0 0}$ \\
\hline Day 2-Day 3 & 1.64 & $\mathbf{0 . 0 3 0}$ \\
\hline Day 3-Day 4 & 0.825 & 0.360 \\
\hline
\end{tabular}

On the other hand, a comparison of the means of total serum bilirubin in the control group only showed a significant decrease of the TSB between day 0 and day $2(p=0.022)$ but no significant decrease has been demonstrated between day 0 and day $1(\mathrm{p}=0.157)$ neither between day 0 and day $3(\mathrm{p}=0.134)$ (Table 2). 
Table 2: Variation of the difference in mean TSB in the control group

\begin{tabular}{|c|c|c|}
\hline & $\begin{array}{c}\text { Mean of the difference } \\
\text { in TSB }\end{array}$ & P Value \\
\hline Day 0-Day 1 & 1.157 & 0.157 \\
\hline Day 0-Day 2 & 3.36 & 0.022 \\
\hline Day 0- Day 3 & 3.50 & 0.134 \\
\hline Day 1- Day 2 & 1.45 & 0.137 \\
\hline Day 2- Day 3 & 1.41 & 0.245 \\
\hline Day 3- Day 4 & 2.45 & 0.189 \\
\hline
\end{tabular}

The duration of hospitalization was not affected by whether the cause of hyperbilirubinemia was $\mathrm{ABO} / \mathrm{Rh}$ incompatibility or not $(\mathrm{p}=0.28)$, neither it was different when comparing the two groups.

\section{Discussion}

Neonatal jaundice is a frequent problem of the newborn with various etiologies. It requires early treatment to prevent complications and the possible accumulation of bilirubin in the white matter of the brain, which leads to irreversible neurological sequelae: kernicterus.

Phototherapy has been the gold standard treatment of neonatal jaundice as it is able to significantly and quickly decrease total serum bilirubin. However, long durations of phototherapy as well as using high wave intensities can have some undesirable effects in newborns such as hyperthermia, erythematous rashes, burns and diarrhea. (Ennever et al. 1987)

Probiotics have recently been an object of interest in this regard because of their ability to increase excretion of bilirubin and therefore decrease neonatal jaundice more rapidly when coupled with phototherapy.(W., H., and T. 2015) (Torkaman et al. 2016) (Demirel et al. 2013)

They have proven to be very beneficial for intestinal motility and integrity and for the modulation of the immune system and have been used in studies on newborns with jaundice as an adjuvant to phototherapy, in which they have contributed to a quicker and more significant decrease in total serum bilirubin with no documented side effects.

In Demirel et al's study in 2012 in Turkey, preterm newborns with hyperbilirubinemia were divided into two groups:

The control group (98 neonates) received breast milk or formula without a probiotic, and the treatment group ( 81 neonates) received breast milk or formula with the probiotic S. boulardii. The obtained results demonstrated a significant decrease in the duration of hospitalization in the group receiving the probiotic when compared with the control group $(1.9 \pm 0.86$ days in the treatment group compared with $2.6 \pm 0.9$ days in the control group with a $p$ value of 0.000).(Demirel et al. 2013) 
Liu W. et al in 2015 divided 68 term neonates with jaundice into two groups, where the first group received phototherapy along with phenobarbital, and the second group received phototherapy with the probiotic "bifid triple viable". The decrease of serum bilirubin was more significant at day 4 $(\mathrm{p}=0.002)$ and day $7(\mathrm{p}=0.001)$ in the group that received the probiotic.(W., H., and T. 2015)

In Torkaman M. et al's study in 2015, the objective was to determine the effect of administration of probiotic on the duration of hospitalization. 92 neonates at term with jaundice were divided into two groups, where the treatment group received the probiotic "Prokid" and the control group received a placebo. Their results demonstrated a significant decrease in the duration of hospitalization $(\mathrm{p}=0.001)$ in the treatment group.(Torkaman et al. 2016)

Our study showed significant difference between control and intervention groups especially in the first three days of treatment weather jaundice is due to incompatibility or not.

Although phototherapy has a triple mechanism of action leading to the conversion of bilirubin into lamirubin preventing the deposition of bilirubin in the tissues, we have demonstrated that the conjunctive use of probiotic L. reuteri has an effect of accelerating the intestinal motility and the reabsorption of biliary acids in the entero-hepatic circulation thereby accelerating the excretion of bilirubin and preventing its toxic accumulation.

\section{Conclusion}

The treatment of neonatal jaundice has been approached using several medical treatments, which have been banned, from the market. This problem has encouraged researchers to find new therapies that have the capacity to decrease bilirubin with minimal side effects.

In our study, we have demonstrated that using a probiotic in conjunction with phototherapy can lead to a significant decrease in serum bilirubin in newborns, especially during the first two days of treatment. However, it did not affect the duration of hospitalization . Larger scale studies should be done to determine if these results could be duplicated.

Authors declare no conflict of interest

\section{References:}

1. Alcayd, Sabine. 2008. "Ictères Du Nouveau-Né." : 1-11.

2. Chang, Hung-Yang et al. 2017. "Multiple Strains Probiotics Appear to Be the Most Effective Probiotics in the Prevention of Necrotizing Enterocolitis and Mortality: An Updated Meta-Analysis." Plos One 12(2): e0171579. http://dx.plos.org/10.1371/journal.pone.0171579.

3. Crigler, J. F., and N. I. Gold. 1969. "Effect of Sodium Phenobarbital on Bilirubin Metabolism in an Infant with Congenital, Nonhemolytic, 
Unconjugated Hyperbilirubinemia, and Kernicterus." Journal of Clinical Investigation 48(1): 42-55.

4. Cuello-Garcia, Carlos a. et al. 2015. "Probiotics for the Prevention of Allergy: A Systematic Review and Meta-Analysis of Randomized Controlled Trials." Journal of Allergy and Clinical Immunology: 1-10. http://linkinghub.elsevier.com/retrieve/pii/S0091674915006363.

5. Cui, Shusheng, and Ying Hu. 2012. "Multistrain Probiotic Preparation Significantly Reduces Symptoms of Irritable Bowel Syndrome in a Double-Blind Placebo-Controlled Study." International Journal of Clinical and Experimental Medicine 5(3): 238-44.

6. Demirel, G, I H Celik, O Erdeve, and U Dilmen. 2013. "Impact of Probiotics on the Course of Indirect Hyperbilirubinemia and Phototherapy Duration in Very Low Birth Weight Infants." J Matern Fetal Neonatal Med 26(2): 215-18. http://www.ncbi.nlm.nih.gov/pubmed/22937831.

7. Ennever, J. F., A. T. Costarino, R. A. Polin, and W. T. Speck. 1987. "Rapid Clearance of a Structural Isomer of Bilirubin during Phototherapy." Journal of Clinical Investigation 79(6): 1674-78.

8. Forsberg, A., T. R. Abrahamsson, B. Björkstén, and M. C. Jenmalm. 2013. "Pre- and Post-Natal Lactobacillus Reuteri Supplementation Decreases Allergen Responsiveness in Infancy." Clinical and Experimental Allergy 43(4): 434-42.

9. Gogineni, Vijaya K, Lee E Morrow, and Mark a Malesker. 2013. "Probiotics \& Health Probiotics: Mechanisms of Action and Clinical Applications Immune Modulation." Journal of Probioitc and Health 1(1): $1-11$.

10. Harmsen, Hermie J M. 2016. "Microbiota of the Human Body." 902: 95-108. http://link.springer.com/10.1007/978-3-319-31248-4.

11. Huang, Wendong, Jun Zhang, and David D. Moore. 2004. "A Traditional Herbal Medicine Enhances Bilirubin Clearance by Activating the Nuclear Receptor CAR." Journal of Clinical Investigation 113(1): 137-43.

12. Issa, Iyad, and Rami Moucari. 2014. "Probiotics for AntibioticAssociated Diarrhea: Do We Have a Verdict?" World Journal of Gastroenterology 20(47): 17788-95.

13. Kwon, John, and Richard Farrell. 2003. "Probiotics and Inflammatory Bowel Disease." BioDrugs: clinical immunotherapeutics, biopharmaceuticals and gene therapy 17(3): 179-86.

14. Mi, Guo Lin et al. 2015. "Effectiveness of Lactobacillus Reuteri in Infantile Colic and Colicky Induced Maternal Depression: A Prospective Single Blind Randomized Trial." Antonie van Leeuwenhoek, International Journal of General and Molecular 
Microbiology 107(6): 1547-53. http://dx.doi.org/10.1007/s10482015-0448-9.

15. Mutombo, André Kabamba et al. 2014. "Ict??res Pathologiques Du Nouveau-N?? ?? L'h??pital Bonzola de Mbuji-Mayi, R??publique D??mocratique Du Congo." The Pan African medical journal 19: 302.

16. Partty, Anna et al. 2015. "Probiotic Lactobacillus Rhamnosus GG Therapy and Microbiological Programming in Infantile Colic: A Randomized, Controlled Trial.” Pediatric Research 78(4): 470-75.

17. Porter, M. L., and B. L. Dennis. 2002. "Hyperbilirubinemia in the Term Newborn." American Family Physician 65(4): 599-606.

18. Roberts, Donna M., Michael Ostapchuk, and James G. O'Brien. 2004. "Infantile Colic." American Family Physician 70(4): 735-42.

19. S, Hemaiswarya, R Raja, R Ravikumar, and Isabel S Carvalho. 2013. "BRAZILIAN ARCHIVES OF BIOLOGY AND TECHNOLOGY Mechanism of Action of Probiotics." Arch. Biol. Technol. v 56156(1): 113-19.

20. Seyyedrasooli, a et al. 2014. "Effect of Vimala Massage on Physiological Jaundice in Infants: A Randomized Controlled Trial." Journal of Caring Sciences 3(3): 165-73.

21. Silverman, Michael A., Liza Konnikova, and Jeffrey S. Gerber. 2017. "Impact of Antibiotics on Necrotizing Enterocolitis and AntibioticAssociated Diarrhea." Gastroenterology Clinics of North America 46(1):

61-76. http://www.ncbi.nlm.nih.gov/pubmed/28164853\%0Ahttp://linkinghu b.elsevier.com/retrieve/pii/S0889855316300851.

22. Torkaman, Mohammad et al. 2016. "The Effect of Probiotics on Reducing Hospitalization Duration in Infants With Hyperbilirubinemia." : 1-6.

23. W., Liu, Liu H., and Wang T. 2015. "Therapeutic Effects of Probiotics on Neonatal Jaundice." Pakistan Journal of Medical Sciences 31(5): no pagination. http://www.pjms.com.pk/index.php/pjms/article/download/7921/3581 \%5Cnhttp://ovidsp.ovid.com/ovidweb.cgi?T=JS\&PAGE=reference\& $\mathrm{D}=\mathrm{emed} 13 \& \mathrm{NEWS}=\mathrm{N} \& \mathrm{AN}=2015389319$. 